\title{
Some Aspects of the Influence of Population Diversity on the Performance of Differential Evolution
}

\author{
João Claudio Chamma Carvalho \\ Faculty of Computation \\ Federal University of Pará \\ Castanhal, Brazil \\ joaochamma@ufpa.br
}

\author{
Kalef Levy de Lima Pinto \\ Applied Electromagnetism Laboratory \\ Federal University of Pará \\ Belém, Brazil \\ CNPq scholarship holder - Brazil \\ kalefllpinto@gmail.com
}

\author{
Roberto Célio Limão Oliveira \\ Faculty of Computer Engineering \\ Federal University of Pará \\ Belém, Brazil \\ limao@ufpa.br
}

\begin{abstract}
This paper presents a study about some aspects of the influence of population diversity on the performance of the Differential Evolution (DE) technique. In order to accomplish this, three different variants of this technique, Standard DE, JADE, and SaDE, are tested through five unconstrained benchmark functions, and the performance results are analyzed and discussed by associating variations in the population diversity with changes in the evolution of the best solution over the generations. The objective of this work is to investigate the pattern of diversity behavior throughout the optimization process through graphs results and, then, evaluate how sensitive is the technique performance when associated with the population diversity behavior. Regarding the results, JADE has achieved the best performance for almost all the benchmark functions tested. Furthermore, the graphs indicate the performance of the variants is highly influenced by how the algorithms control the diversity throughout the optimization process and that a high average diversity is not always a guarantee of better performance. This work can assist the implementation of new operators and strategies, which will permit the Differential Evolution technique to have a better performance.
\end{abstract}

Index Terms-differential evolution, population diversity, exploration, exploitation

\section{INTRODUCTION}

In the last decades, many algorithms have been proposed to find the optimal value of objective functions with real search space [1]-[3]. Among them, stand out the techniques that perform a global search and generate their initial solutions [4]-[6], which are able to find better results when compared to traditional optimization techniques [2], [7], [8]. Among these global optimization techniques, the Differential Evolution (DE) has attracted the interest of researchers in the last decades due to its simplicity and robustness in the search for the global maximum. Such a technique consists of an evolutionary algorithm, which was proposed by R. Storn and K. Price [9]. DE has become one of the most used natureinspired Algorithms in problems of global optimization of real variables [10]. The myriad of applications of this technique in real-world problems successfully demonstrates its excellent performance [11].
The main difference between DE and other well-known nature-inspired algorithms lies in the fact that each vector can mutate with differences in scale between distinct members, tending to adapt to the scales of objective function [12]. In addition to the mutation, the DE has the operators of selection, crossing, and control parameters (mutation factor and crossing probability) that must have adequate values to the problem in order to obtain a better performance [11].

Regarding the works in the literature that attempts to increase the diversity in DE, we can mention the use of subpopulations proposed by Zaharie [13] to achieve the trade-off between exploration and exploitation in the Differential Evolution (DE) technique. Moreover, J. Zhang and A. Sanderson proposed the Adaptive Differential Evolution with Optional External Archive [14], named by JADE. This algorithm implements a new mutation strategy named "DE/current-to-pbest", which is a generalization of the classic "DE / current-to-best" strategy. Another feature of JADE is the use of an external file operation, which consists of a history of information used to direct the progress of the algorithm in order to diversify the population throughout the execution. In addition, JADE also updates the control parameters adaptively. Furthermore, A. Qin and P. Suganthan proposed the Self-adapted Differential Evolution (SaDE) [15], which implements a set of candidate strategies to generate the trial vector. In this variant, both the trial vector generation strategies and their associated control parameter values are gradually adapted using information from previous experiments obtained from previous generations in which promising solutions were found.

The SaDE and JADE algorithms reuse promising information from previous generations, either by reinserting old solutions directly into the population (JADE) or by gradually adapting control parameters according to previous experiences (SaDE) [14], [15]. These techniques reuse information from previous generations and this feature improves diversity by facilitating the search for high-quality solutions, which makes them have an excellent performance when compared to other DE variants [16]-[18]. 
However, what is the expected behavior of the population diversity in DE over the generations in order to maintain a good relationship between exploration and exploitation? With the view to analyzing such behavior in DE variants, this work investigates the influence of the variation of diversity over the generations on the DE's performance and, then, evaluates how sensitive is this technique performance when associated with the population diversity behavior. To accomplish this study, the standard DE, in addition to the JADE and SaDE variants, are applied to the optimization of five benchmark functions: Ackley, Rastrigin, Griewank, Schaffer e Schwefel. The results concerning the evolution and diversity graphs are analyzed and their patterns are associated with each other to find strong relationships and verify what are the diversity behavior characteristics that improve the DE performance.

\section{DIVERSITY}

The definition of population diversity in nature-inspired algorithms refers to how individuals in the population are distributed on the search space [19]. Different ways of measuring population diversity have been proposed. Two of them frequently used in GAs are the population diversity based on Hamming distances [20], [21] and the Euclidean distance, which is common for distance estimation with real-coded genes [11]. The latter was used by B. Ginley et. al. [22] and R. Ursem [23], which proposed an algorithm called DiversityGuided Evolutionary Algorithm (DGEA) that measures the diversity using a mechanism to detect the exploration and exploitation phases. In order to accomplish such a mechanism, the measurement technique must be robust concerning population size, the dimensionality of the problem, and the limit of each variable [23]. Because of these three characteristics, the Euclidean distance to the midpoint used by Ursem in the DGEA was chosen to measure the diversity, then for comparison purposes, this type of diversity was used in all DE variants implemented in this article. The equation (1) is a brief description of such a measure of diversity:

$$
\operatorname{diversity}(P)=\frac{1}{|L| \cdot|P|} \cdot \sum_{i=1}^{|P|} \sqrt{\sum_{j=1}^{N}\left(S_{i, j}-\overline{S_{j}}\right)^{2}}
$$

where $|L|$ is the length of the diagonal in the search space $S \subseteq \Re^{N}, P$ is the population, $|P|$ is the population size, $N$ is the dimension of the problem, $S_{i, j}$ is the $j t h$ value of the $i t h$ individual, and $\overline{S_{j}}$ is the $j t h$ value of the average point $S$.

It is known that population diversity plays an important role in the performance of nature-inspired algorithms [11]. Many researchers believe that the efficiency of a nature-inspired algorithm is influenced by the appropriate ratio between exploitation and exploration [24]. One can understand the exploration process as the process of visiting new regions of the search and exploitation space as the process of visiting regions of the search space that are part of the neighborhood of the points visited previously. On the basis of these concepts, it is noted that there is a close connection between maintaining an adequate balance of exploration/exploitation and the behavior of diversity over generations. Adding to this the fact that measuring and controlling exploitation and exploration is still a major challenge [24], it is necessary to evaluate these effects from the behavior of diversity. For this reason, it is important to understand how sensitive the performance of global optimization algorithms is when they undergo variations in population diversity.

\section{BENCHMARK FUNCTIONS USED IN THE SIMULATIONS}

The three DE variants, Standard DE, JADE and SaDE, were tested using five unconstrained benchmark functions, which are: Ackley, Rastrigin, Griewank, Schaffer e Schwefel. All these functions have been adapted to be maximization problems and present different characteristics and spatial shapes. In table I are shown the benchmark functions, with their respective maximum values (Max), dimensionality (Dim), search space range (Range) and other characteristics (Charac), including information about multimodality and linearity.

TABLE I

LIST OF BENCHMARK FUNCTIONS

\begin{tabular}{|c|c|c|c|c|}
\hline Name & Dim & Max & Range & Charac \\
\hline Ackley & 10 & 22.314 & {$[-32,32]$} & $\begin{array}{c}\text { Continuous } \\
\text { Differentiable } \\
\text { Non-separable } \\
\text { Non-convex } \\
\text { Multimodal }\end{array}$ \\
\hline Rastrigin & 10 & 403.53 & {$[-5.12,5.12]$} & $\begin{array}{c}\text { Continuous } \\
\text { Differentiable } \\
\text { Separable } \\
\text { Convex } \\
\text { Multimodal }\end{array}$ \\
\hline Griewank & 10 & 900 & {$[-600,600]$} & $\begin{array}{c}\text { Continuous } \\
\text { Differentiable } \\
\text { Non-separable } \\
\text { Non-convex } \\
\text { Multimodal }\end{array}$ \\
\hline Schaffer & 10 & 1 & {$[-30,30]$} & $\begin{array}{c}\text { Continuous } \\
\text { Differentiable } \\
\text { Non-separable } \\
\text { Non-convex } \\
\text { Unimodal }\end{array}$ \\
\hline Schwefel & 10 & 8380 & {$[-500,500]$} & $\begin{array}{c}\text { Continuous } \\
\text { Differentiable } \\
\text { Separable } \\
\text { Non-convex } \\
\text { Multimodal }\end{array}$ \\
\hline
\end{tabular}

For each test function, the averaged results of 50 independent runs with the same initial population were recorded. The diversity and evolution curves represent, therefore, the average values of such 50 runs.

The main objective of the experiment is to associate variations in the behavior of diversity with the performance of DE variants. It is expected in future works that this association allows the designer to configure the algorithm, introducing mechanisms of diversity control to improve the performance of the DE. The descriptive statistics of the results are shown in table II, which contains (for each experiment performed and considering all generations) the following values: maximum 
reached value (named by Max in table II ), average diversity (named by AveDiv in table II) and standard deviation of diversity (named by StdDiv in table II).

\section{ANALYSIS AND RESUlTS}

As mentioned in the introduction, this section is dedicated to the presentation and discussion of the graphs related to the simulation results of the Differential Evolution variants applied to the optimization of five benchmark functions. In order to investigate the influence of population diversity on the performance of such a technique, the behavior of each evolution graph is associated with the variations in the respective diversity graph throughout the generations. Furthermore, the results in the table II are used as additional quantitative information to aggregate in the analysis. Finally, it is worth mentioning that, for all the simulations, the stopping criterion is the number of generations; however, a different number of total generations is used for each benchmark function, because the algorithms reach the stabilization in distinct moments depending on the function to be optimized.

Figures from 1 to 5 show the evolution and diversity curves of the techniques JADE, SaDE, and standard DE. In figure 1.(a) we have the graph of the evolution of the techniques (DE variants) for the Ackley function. In figure 1.(a) we can see that the standard DE algorithm has a better initial performance but later is exceeded by the JADE technique and then by the SaDE technique. The standard DE after its abrupt initial growth suffers a stagnation that begins around the 1000th generation. Relating this performance behavior to the diversity graph in figure 1.(b), we see that the diversity of the standard DE drops abruptly to very small values. This lack of smoothness in diversity behavior shows that a better balance is needed between exploitation and exploration within the algorithm. Through the zoom in the image, which is within figure 1.(a), we see that the SaDE overcomes the other two techniques and then suffers a stagnation around the 7000 generation and is finally overtaken by JADE.

The graph of diversity in figure 1.(b) shows that $\mathrm{SaDE}$ shows a slight increase in its diversity at the beginning, which means that its exploration process was more intense and prolonged than that of standard DE, thus justifying why SaDE starts the optimization process with less performance than the other techniques when we look at the evolution graph in figure 1.(a). However, throughout the process, we can see that SaDE presents a better balance during the transition between exploration and exploitation when compared to standard DE.

Still analyzing figure 1.(a) and figure 1.(b) we see that the JADE algorithm presents a more efficient exploration phase, which is characterized by the increase of diversity and good evolution performance. This technique has also an excellent exploitation phase, which improves its ability to refine the solution. This algorithm performed better than the $\mathrm{SaDE}$ algorithm and obtained the best result among the three techniques. It is important to highlight a particular characteristic in JADE diversity behavior for the Ackley function. Among all functions, this was the only one in which JADE

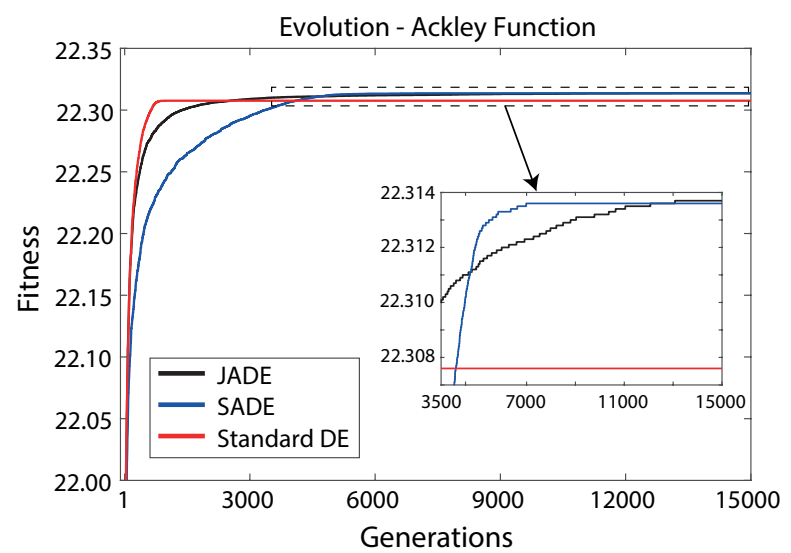

(a)

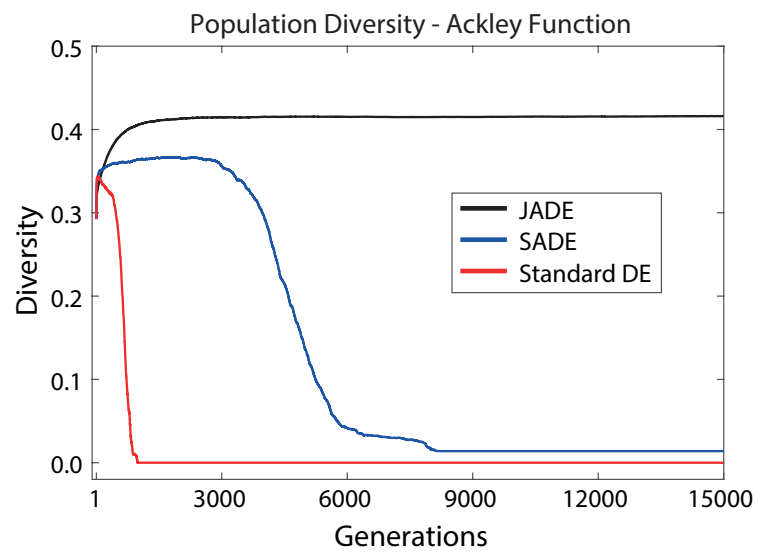

(b)

Fig. 1. Evolution of the best solution (a) and population diversity (b). Both graphs refer to Ackley function and compare the techniques JADE, SaDE and Standard DE.

maintained its diversity with high values until the end of the optimization, without presenting any drop at any point in the evolutionary process. We believe that this is closely related to the characteristics of the Ackley function.

The tests performed with the Rastrigin, Griewank, and Schwefel functions, which are shown in figures 2, 3 and 4 , respectively, present interesting patterns and allow us to extract some interesting conclusions. For these three functions, the JADE technique obtained the best performance when we analyzed the evolution graphs (figure 2.(a), figure 3.(a), figure 4.(a)), so that the performance of this technique surpasses the others two in the last generations of the process similar to what happened in the Ackley function. This is related to the graphs of diversities (figure 2.(b), figure 3.(b), figure 4.(b)) it can be seen that the JADE diversity does not fall abruptly at any moment, but shows a slight decrease along with the generations. On the other hand, in these same graphs of diversity one observes at a given moment a sudden drop of the diversity of the techniques SaDE and standard DE. In line with this prominent decline in diversity, there is also a noticeable 
TABLE II

DESCRIPTIVE STATISTICS OF THE RESULTS

\begin{tabular}{|c|c|c|c|c|c|c|c|c|c|}
\hline \multirow{3}{*}{ Ackley } & \multicolumn{3}{|c|}{ Standard DE } & \multicolumn{3}{|c|}{ JADE } & \multicolumn{3}{|c|}{ SaDE } \\
\hline & $\operatorname{Max}$ & AveDiv & StdDiv & $\operatorname{Max}$ & AveDiv & StdDiv & $\operatorname{Max}$ & AveDiv & StdDiv \\
\hline & $2.231 \mathrm{E}+1$ & $1.454 \mathrm{E}-2$ & $6.370 \mathrm{E}-2$ & $2.231 \mathrm{E}+1$ & $4.126 \mathrm{E}-1$ & $1.064 \mathrm{E}-2$ & $2.231 \mathrm{E}+1$ & $1.247 \mathrm{E}-1$ & $1.484 \mathrm{E}-1$ \\
\hline \multirow{3}{*}{ Rastrigin } & \multicolumn{3}{|c|}{ Standard DE } & \multicolumn{3}{|c|}{ JADE } & \multicolumn{3}{|c|}{ SaDE } \\
\hline & Max & AveDiv & StdDiv & Max & AveDiv & StdDiv & $\operatorname{Max}$ & AveDiv & StdDiv \\
\hline & $4.035 \mathrm{E}+2$ & $6.307 \mathrm{E}-3$ & $2.56 \mathrm{E}-2$ & $4.035 \mathrm{E}+2$ & $3.364 \mathrm{E}-2$ & $2.841 \mathrm{E}-2$ & $4.035 \mathrm{E}+2$ & $8.329 \mathrm{E}-3$ & $2.790 \mathrm{E}-2$ \\
\hline & \multirow{2}{*}{\multicolumn{3}{|c|}{ Standard DE }} & \multirow{2}{*}{\multicolumn{3}{|c|}{ JADE }} & & & \\
\hline \multirow{3}{*}{ Griewank } & & & & & & & \multicolumn{3}{|c|}{ SaDE } \\
\hline & $\operatorname{Max}$ & AveDiv & StdDiv & $\operatorname{Max}$ & AveDiv & StdDiv & $\operatorname{Max}$ & AveDiv & StdDiv \\
\hline & $9.013 \mathrm{E}+2$ & $1.317 \mathrm{E}-3$ & $1.242 \mathrm{E}-2$ & $9.011 \mathrm{E}+2$ & $3.143 \mathrm{E}-3$ & $1.172 \mathrm{E}-2$ & $9.014 \mathrm{E}+2$ & $1.097 \mathrm{E}-3$ & $1.178 \mathrm{E}-2$ \\
\hline \multirow{3}{*}{ Schwefel } & \multicolumn{3}{|c|}{ Standard DE } & \multicolumn{3}{|c|}{ JADE } & \multicolumn{3}{|c|}{ SaDE } \\
\hline & $\operatorname{Max}$ & AveDiv & StdDiv & $\operatorname{Max}$ & AveDiv & StdDiv & $\operatorname{Max}$ & AveDiv & StdDiv \\
\hline & $8.328 \mathrm{E}+3$ & $1.172 \mathrm{E}-2$ & $5.788 \mathrm{E}-2$ & $8.368 \mathrm{E}+3$ & $2.927 \mathrm{E}-1$ & $3.014 \mathrm{E}-2$ & $8.361 \mathrm{E}+3$ & $2.049 \mathrm{E}-2$ & $7.71 \mathrm{E}-2$ \\
\hline \multirow{3}{*}{ Schaffer } & \multicolumn{3}{|c|}{ Standard DE } & \multicolumn{3}{|c|}{ JADE } & \multicolumn{3}{|c|}{ SaDE } \\
\hline & $\operatorname{Max}$ & AveDiv & StdDiv & $\operatorname{Max}$ & AveDiv & StdDiv & Max & AveDiv & StdDiv \\
\hline & $9.975 \mathrm{E}-1$ & $1.862 \mathrm{E}-2$ & $2.231 \mathrm{E}-2$ & $9.797 \mathrm{E}-1$ & $2.553 \mathrm{E}-2$ & $2.149 \mathrm{E}-2$ & $9.975 \mathrm{E}-1$ & $1.351 \mathrm{E}-2$ & $2.054 \mathrm{E}-2$ \\
\hline
\end{tabular}

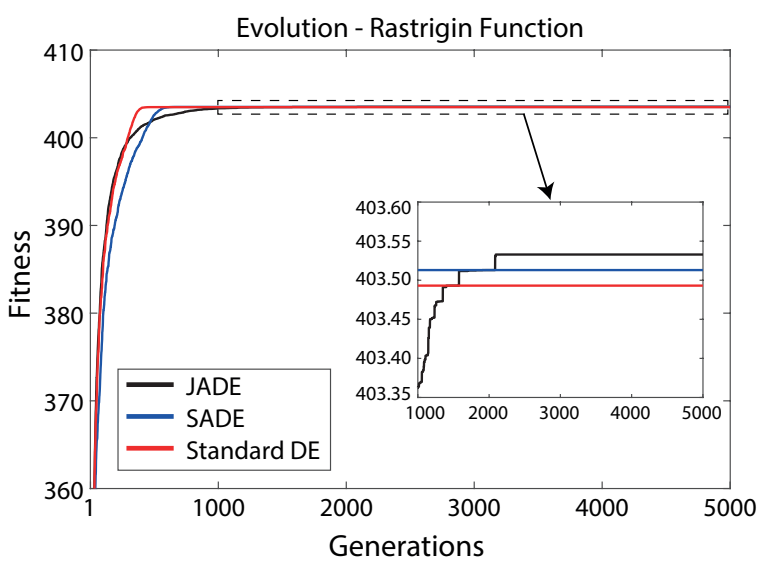

(a)

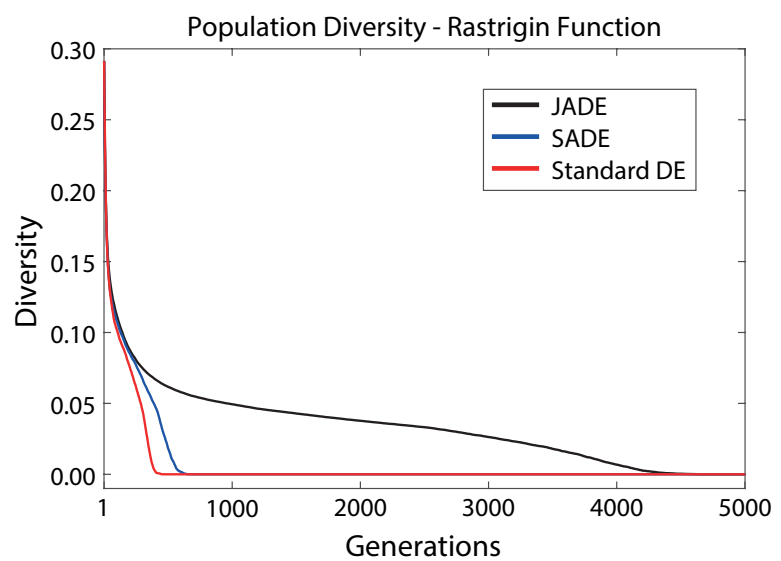

(b)

Fig. 2. Evolution of the best solution (a) and population diversity (b). Both graphs refer to Rastrigin function and compare the techniques JADE, SaDE and Standard DE.

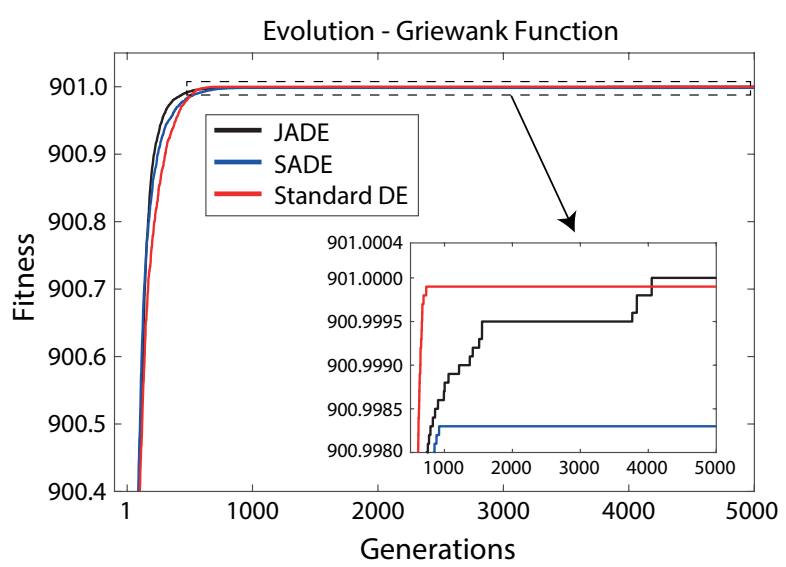

(a)

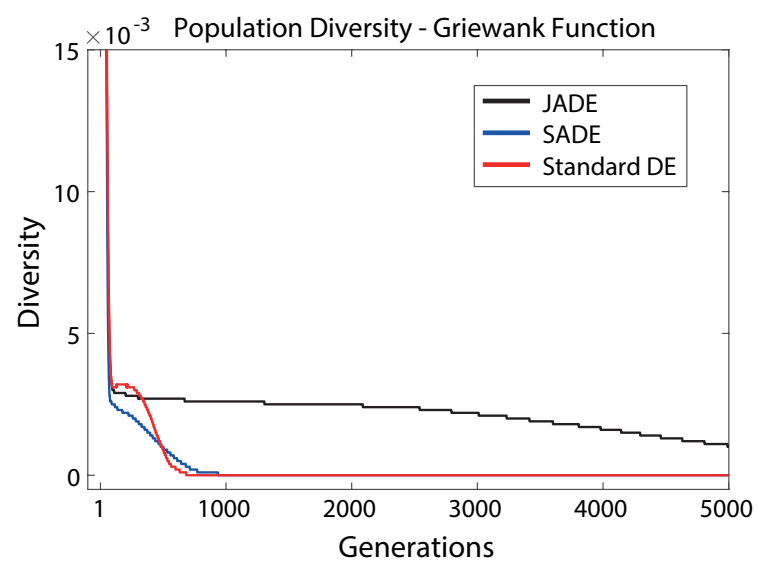

(b)

Fig. 3. Evolution of the best solution (a) and population diversity (b). Both graphs refer to Griewank function and compare the techniques JADE, SaDE and Standard DE. 


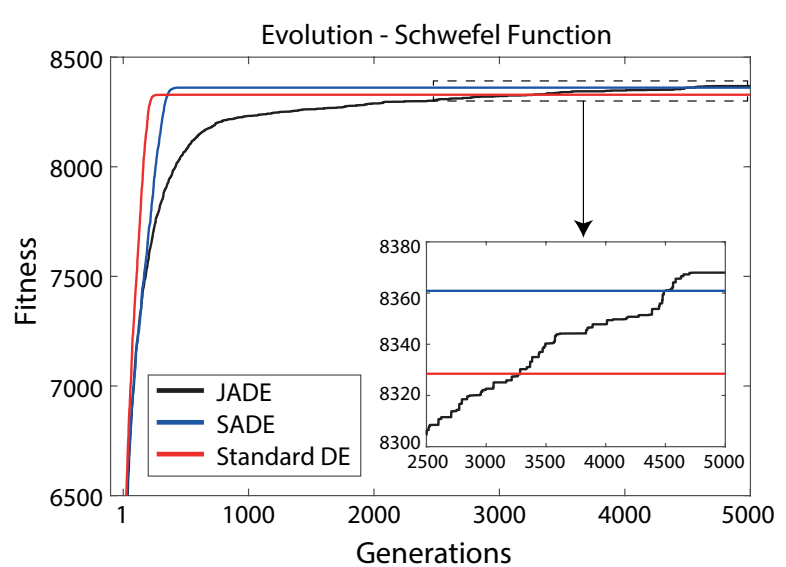

(a)

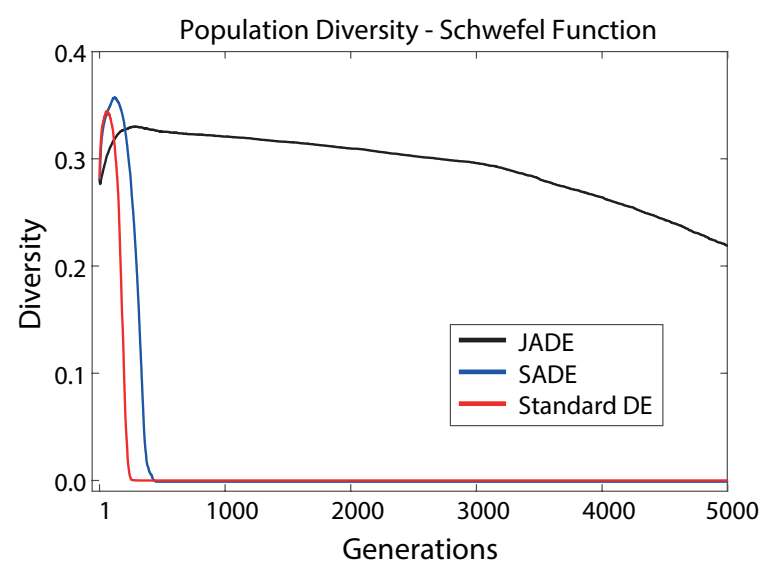

(b)

Fig. 4. Evolution of the best solution (a) and population diversity (b). Both graphs refer to Schwefel function and compare the techniques JADE, SaDE and Standard DE.

stagnation in the SaDE and standard DE evolution graphs for the three functions in question, suggesting that abrupt changes in diversity tend to prejudices the exploitation of algorithms.

The graph of figure 5 shows that, unlike what happened in the other functions, JADE had the worst performance when compared to the other two algorithms. A possible explanation for this may also be found in the JADE diversities behaviors, which initiate a stagnation around the 800th generation, making the technique remain with almost constant diversity in the remaining generations. The results suggest that this stagnation of diversity impaired the exploitation of the algorithm, although the diversity remained high when compared to the other two techniques. This also shows that keeping diversity constant and at high levels throughout the process does not guarantee better performance of the technique. In relation to the other two variations of the $\mathrm{DE}$, it can be seen from figure 5.(b) that, unlike JADE, the SaDE and standard DE diversity decrease moderately throughout the final generations. This characteristic possibly contributed to the fact that these two

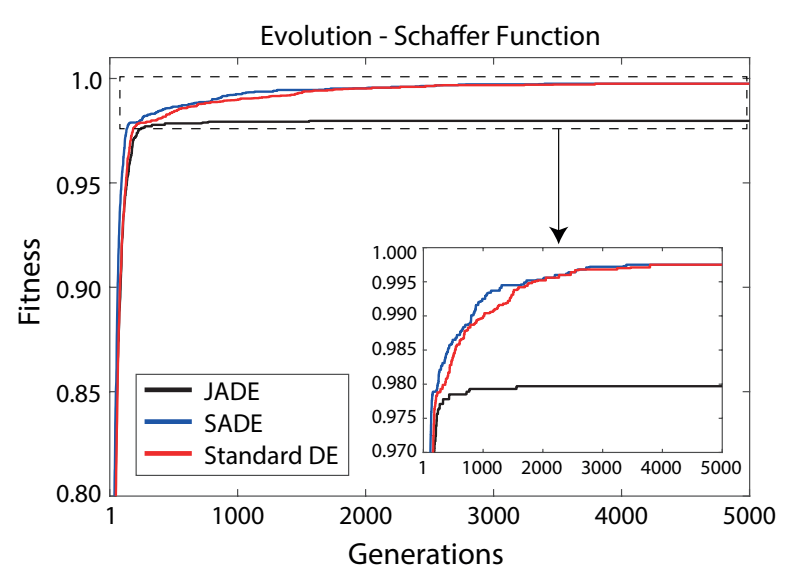

(a)

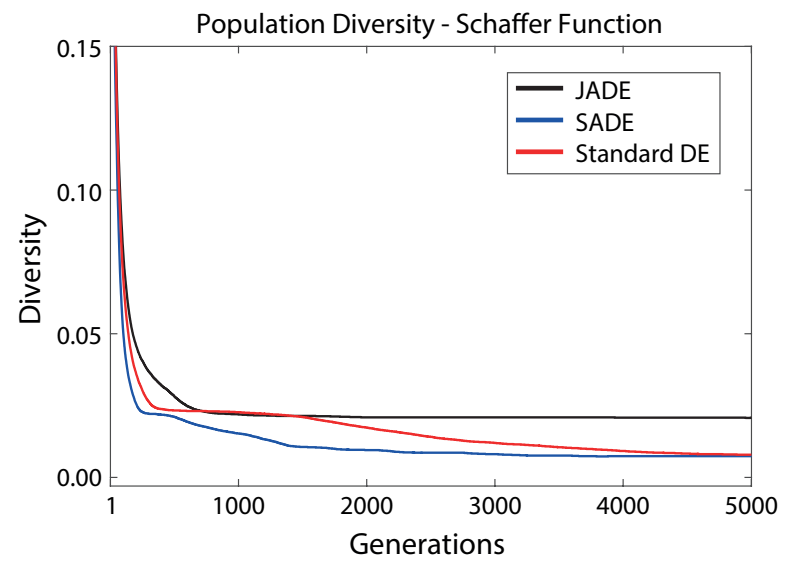

(b)

Fig. 5. Evolution of the best solution (a) and population diversity (b). Both graphs refer to Schaffer function and compare the techniques JADE, SaDE and Standard DE.

techniques performed better than JADE, since it favored more robust exploitation, increasing the power of refinement of these algorithms, similar to what happened to JADE in the functions Rastrigin, Griewank, and Schwefel. In analyzing the graphs of evolution (figure 2.(a), figure 3.(a) and figure 4.(a)) it is noted that the JADE technique has been able to overcome the other techniques in the last generations, similar to what happened in the Ackley function.

From the graphs of figures 2.(b), figure 3.(b) and figure 4.(b), it is noted that the diversity of JADE does not fall abruptly, but shows a slight decrease over the generations. On the other hand, in these same graphs of diversity, it is observed that the techniques $\mathrm{SaDE}$ and standard DE show a sudden drop in diversity. This sharp drop in diversity is associated with visible stagnation in the SaDE and standard DE evolution charts for the Rastrigin, Griewank, and Schwefel functions, suggesting that abrupt changes in diversity tend to undermine the exploitation of algorithms. 


\section{CONClusion}

In this work, the importance of diversity behavior in the performance of Differential Evolution algorithms was investigated. Three DE variants were used: standard DE, SaDE, and JADE. The tests showed that regardless of the strategies adopted by the techniques to find the global optimum, their performance is strongly influenced by the way they control diversity throughout the optimization process. The results showed that the best performance was achieved by JADE, except for the Schaffer function, where JADE was the worst. Also, for some results, JADE did not present the best performance during the beginning of the optimization, although this variant outperformed the other two in the later generations. This pattern suggests that it is not enough to the technique to find the best solution, but accomplish that as quickly as possible.

As verified in the tests, a high average diversity is not always a guarantee of better performance. It is important that the technique also has mechanisms capable of promoting variations at appropriate levels to accomplish a suitable balance between the exploration and exploitation phases. In this context, the diversity graphs are a useful tool since they permit the developers to associate the performances of the variants with the population diversity behavior. This association benefits the development of novel mechanisms to not only increase the diversity, however, are also able to decide at what moment and to what level such a variation should occur. The implementation of new operators and mechanisms based on the behavior of diversity curves to improve the performance of the DE in the search for the global optimum will be the focus of future works.

\section{REFERENCES}

[1] G. S. G. Beveridge and R. S. Schechter, Optimization : theory and practice. New York; Maidenhead : McGraw-Hill, 1970.

[2] S. Chandra and A. Mehra, Numerical Optimization with Applications. Alpha Science International, 2009.

[3] D. Greiner, B. Galván, J. Périaux, N. Gauger, K. Giannakoglou, and G. Winter, Advances in Evolutionary and Deterministic Methods for Design, Optimization and Control in Engineering and Sciences, ser Computational Methods in Applied Sciences. Springer International Publishing, 2014, vol. 36.

[4] R. Horst, P. Pardalos, and N. Van Thoai, Introduction to Global Optimization, ser. Nonconvex Optimization and Its Applications. Springer US, 2000, vol. 48.

[5] L. Liberti, "Introduction to global optimization," Lecture of Ecole Polytechnique, Palaiseau F, vol. 91128, p. 43, 2008.

[6] E. Hendrix and B. Tóth, Introduction to Nonlinear and Global Optimization, ser. Springer Optimization and Its Applications. Springer New York, 2010, vol. 37.

[7] I. Zelinka, V. Snasael, and A. Abraham, Handbook of Optimization: From Classical to Modern Approach, ser. Intelligent Systems Reference Library. Springer Berlin Heidelberg, 2012, vol. 38.

[8] S. Luke, Essentials of Metaheuristics, 2nd ed. Lulu, 2013.

[9] R. Storn and K. Price, "Differential evolution - a simple and efficient heuristic for global optimization over continuous spaces," Journal of Global Optimization, vol. 11, no. 4, pp. 341-359, Dec 1997.

[10] K. Price, R. M. Storn, and J. A. Lampinen, Differential Evolution: A Practical Approach to Global Optimization (Natural Computing Series). Secaucus, NJ, USA: Springer-Verlag New York, Inc., 2005.

[11] M. Yang, C. Li, Z. Cai, and J. Guan, "Differential evolution with auto-enhanced population diversity," IEEE Transactions on Cybernetics, vol. 45, no. 2, pp. 302-315, Feb 2015
[12] S. Das, S. Subhra Mullick, and P. Suganthan, "Recent advances in differential evolution - an updated survey," Swarm and Evolutionary Computation, vol. 27, pp. 1-30, 022016.

[13] D. Zaharie, "Control of population Diversity and Adaption in Differential Evolution Algorithms," 9th International Conference on Soft Computing, pp. 41-46, 2003.

[14] J. Zhang and A. C. Sanderson, "Jade: Adaptive differential evolution with optional external archive," IEEE Transactions on Evolutionary Computation, vol. 13, no. 5, pp. 945-958, Oct 2009.

[15] A. K. Qin and P. N. Suganthan, "Self-adaptive differential evolution algorithm for numerical optimization," in 2005 IEEE Congress on Evolutionary Computation, vol. 2, Sept 2005, pp. 1785-1791 Vol. 2.

[16] S. W. Mahfoud, "Niching methods for genetic algorithms," Ph.D. dissertation, Champaign, IL, USA, 1995, uMI Order No. GAX95-43663.

[17] M. Lozano, F. Herrera, and J. R. Cano, "Replacement strategies to preserve useful diversity in steady-state genetic algorithms," Information Sciences, vol. 178, no. 23, pp. 4421 - 4433, 2008, including Special Section: Genetic and Evolutionary Computing.

[18] C. Segura, C. A. C. Coello, E. Segredo, and A. H. Aguirre, "A novel diversity-based replacement strategy for evolutionary algorithms," IEEE Transactions on Cybernetics, vol. 46, no. 12, pp. 3233-3246, Dec 2016.

[19] R. W. Morrison and K. A. De Jong, "Measurement of population diversity," in Artificial Evolution, P. Collet, C. Fonlupt, J.-K. Hao, E. Lutton, and M. Schoenauer, Eds. Berlin, Heidelberg: Springer Berlin Heidelberg, 2002, pp. 31-41.

[20] K. Q. Zhu, "A diversity-controlling adaptive genetic algorithm for the vehicle routing problem with time windows," in Proceedings. 15th IEEE International Conference on Tools with Artificial Intelligence, Nov 2003, pp. 176-183.

[21] H. Shimodaira, "A diversity-control-oriented genetic algorithm (dcga): performance in function optimization," in Proceedings of the 2001 Congress on Evolutionary Computation (IEEE Cat. No.01TH8546), vol. 1, 2001, pp. 44-51 vol. 1.

[22] B. M. Ginley, J. Maher, C. O'Riordan, and F. Morgan, "Maintaining healthy population diversity using adaptive crossover, mutation, and selection," IEEE Transactions on Evolutionary Computation, vol. 15, no. 5, pp. 692-714, Oct 2011.

[23] R. K. Ursem, "Diversity-guided evolutionary algorithms," in Parallel Problem Solving from Nature - PPSN VII, J. J. M. Guervós, P. Adamidis, H.-G. Beyer, H.-P. Schwefel, and J.-L. FernándezVillacañas, Eds. Berlin, Heidelberg: Springer Berlin Heidelberg, 2002, pp. $462-471$.

[24] M. Črepinšek, S.-H. Liu, and M. Mernik, "Exploration and exploitation in evolutionary algorithms: A survey," ACM Comput. Surv., vol. 45, no. 3, pp. 35:1-35:33, Jul. 2013. 